\title{
Médecine personnalisée en oncologie digestive : vers de nouveaux horizons
}

\author{
F. Bibeau \\ (C) Springer-Verlag France 2014
}

L'année 2007 et la mise en place des plateformes d'oncogénétique somatique des tumeurs avaient marqué un tournant en oncologie digestive. Pour la première fois un test moléculaire, facilement réalisable, permettait, pour des patients atteints de cancer colorectal métastatique, d'être mieux sélectionnés pour l'accès à des thérapies ciblées. Il s'agissait de la détermination du statut du gène KRAS dans le cadre de la prescription d'anticorps ciblant l'Epidermal Growth Factor Receptor (EGFR), en l'occurrence le Panitumumab (Vectibix $\left.^{\circledR}\right)[1,2]$. Les mutations des codons 12 et 13 , situés dans l'exon 2 du gène $K R A S$, lorsqu'elles étaient présentes, entrainaient en effet une résistance constante à cette thérapie ciblée, contre-indiquant de ce fait sa prescription [1,2]. Dans la foulée cette même restriction s'est logiquement appliquée au Cetuximab $\left(\right.$ Erbitux $\left.^{\circledR}\right)$, l'autre anticorps monoclonal disponible dans cette situation clinique [2,3]. Cependant les mutations des codons 12 et 13 du gène $K R A S$, recensées dans $40 \%$ des cancers colorectaux, permettent seulement d'identifier des patients résistants. Parmi les $60 \%$ de patients ne présentant pas de tumeurs KRAS mutées (dites « wild-type » ou " sauvages »), la moitié seulement va s'accompagner de réponse objective. L'enjeu de l'après $K R A S$ était donc de démembrer cette population hétérogène, afin de rendre plus efficace l'utilisation des anticorps monoclonaux anti-EGFR.

De la même façon que 2007 a constitué une étape-clé dans la personnalisation des traitements, on peut dire que 2013 renforce le concept de médecine de précision. Plusieurs études ont en effet montré que des mutations supplémentaires à celles des codons 12 et 13 du gène KRAS s'accompagnent également de résistance aux anticorps anti-EGFR [4-7]. Il s'agit de mutations affectant les codons 61 (situé sur l'exon 3), 117 et 146 (situés sur l'exon 4) du gène $K R A S$ et les codons 12,13, 61, 117 et 146 du gène $N R A S$, situés sur les mêmes exons. L'ensemble de ces nouvelles mutations de résistance est présente dans environ $17 \%$ de la population initialement définie comme $K R A S$ sauvage, c'est-à-dire non

\section{F. Bibeau $(\square)$}

Service de pathologie, Institut du Cancer de Montpellier,

34298 Montpellier

e-mail : frederic.bibeau@icm.unicancer.fr mutée pour les codons 12 et 13 . Ces éléments permettent d'identifier maintenant des tumeurs dites " super wildtype » ou «RAS sauvages », c'est-à-dire sans mutations supplémentaires des gènes $K R A S$ et $N R A S$, précédemment mentionnées. Rapportée à la population globale, les tumeurs $R A S$ mutées représentent maintenant $50 \%$ des effectifs, modifiant le ratio précédent $40 \%$ de tumeurs KRAS mutées/60\% de tumeurs $K R A S$ sauvages.

L'étude la plus emblématique pour illustrer l'impact de ces mutations est certainement celle parue dans New England Journal of Medicine en septembre 2013 et coordonnée par Jean-Yves Douillard [4]. Cette étude a reposé sur l'analyse rétrospective et complémentaire du statut mutationnel des tumeurs KRAS sauvages de l'essai PRIME [8]. Cet essai de phase III avait démontré un gain en termes de survie sans progression d'une chimiothérapie par FOLFOX plus panitumumab vs FOLFOX seul en première ligne métastatique, aboutissant à l'autorisation de mise sur le marché de cet anticorps monoclonal dans cette situation clinique. Dans l'analyse complémentaire de l'essai PRIME, $17 \%$ de mutations $R A S$ supplémentaires ont été identifiées. Les tumeurs " super wild-type $R A S$ » étaient associées à une supériorité $\mathrm{du}$ traitement par FOLFOX plus panitumumab vs FOLFOX en survie sans progression ( 10,1 mois vs 7,9 mois, $p=0.004)$, avec des médianes plus élevées que celles objectivées dans l'essai PRIME initial reposant sur le seul statut KRAS $(9,6$ mois vs 8 mois, $\mathrm{p}=0.004)$. Par ailleurs, cette supériorité était également objectivée en survie globale $(25,8$ mois vs 20,2 mois, $p=0.009$ ), alors que le bénéfice n'était pas significatif avec les premières données de l'essai PRIME (23,9 mois vs 19,7 mois, $p=0.07$ ). Enfin, il faut souligner que l'existence d'une mutation RAS était associée à un effet délétère du FOLFOX plus panitumumab vs FOLFOX seul, tant en survie sans progression $(7,3$ mois vs 8,7 mois, $\mathrm{p}=0.008)$ que globale $(15,5$ mois vs 18,7 mois, $\mathrm{p}=0.04)$. Cet effet délétère entre oxaliplatine et anti-EGFR avait déjà été noté chez les patients ayant des tumeurs KRAS mutées, dans l'essai OPUS, comparant en première ligne métastatique FOLFOX plus cetuximab vs FOLFOX seul [9].

Au total, la sélection des patients « super wild-type » permet d'obtenir une efficacité optimale du panitumumab en 
première ligne de traitement des cancers colorectaux métastatiques, dépassant 10 mois en survie sans progression et 24 mois en survie globale. Le concept des « super wildtype » est également confirmé dans l'étude de phase II «PEAK» comparant FOLFOX plus panitumumab vs FOLFOX plus bevacizumab et dans l'étude « FIRE III » comparant le cetuximab vs bevacizumab en première ligne en association avec du FOLFIRI $[5,10]$.

Quelle est la conséquence de ces données biologiques? Le rôle des plateformes d'oncogénétique va s'étoffer, en ayant pour mission d'établir un statut $R A S$ précis, et non plus simplement $K R A S$, dans des délais compatibles avec une prise en charge adaptée [2]. Il s'agit là de données incontournables dans la stratégie thérapeutique du cancer colorectal en première ligne métastatique, devant aboutir à identifier les patients pouvant bénéficier au mieux des thérapies ciblées anti-EGFR et à écarter ceux pour qui aucun avantage n'existe, ou pour lesquels un effet délétère, en cas de mutation $R A S$ et d'association avec l'oxaliplatine, peut survenir. C'est tout l'enjeu de la médecine de précision dans un contexte médico-économique de plus en plus exigeant.

\section{Références}

1. Amado RG, Wolf M, Peeters M, et al (2008). Wild-type KRAS is required for panitumumab efficacy in patients with metastatic colorectal cancer. J Clin Oncol 26:1626-34

2. Bonnes pratiques pour la recherche à visée théranostique de mutations somatiques dans les tumeurs solides. Institut national du cancer. http://www.e-cancer.fr/soins/plates-formes-hospitalieres de génétique moléculaire
3. Van Cutsem E, Köhne CH, Láng I, et al (2011). Cetuximab plus irinotecan, fluorouracil, and leucovorin as first-line treatment for metastatic colorectal cancer: updated analysis of overall survival according to tumor KRAS and BRAF mutation status. J Clin Oncol 29:2011-9

4. Douillard JY, Oliner KS, Siena SJ, et al (2013). PanitumumabFOLFOX4 treatment and RAS mutations in colorectal cancer. N Engl J Med 369:1023-34

5. Schwartzberg LS, Rivera F, Karthaus M, et al (2013). Analysis of KRAS/NRAS mutations in PEAK: A randomized phase II study of FOLFOX6 plus panitumumab (pmab) or bevacizumab (bev) as first-line treatment (tx) for wild-type (WT) KRAS (exon 2) metastatic colorectal cancer (mCRC). J Clin Oncol 31 (Suppl. abstr 3631)

6. Peeters M, Oliner KS, Parker A, et al (2013). Massively parallel tumor multigene sequencing to evaluate response to panitumumab in a randomized phase III study of metastatic colorectal cancer. Clin Cancer Res 19:1902-12

7. Patterson SD, Peeters M, Siena S, et al (2013). Comprehensive analysis of KRAS and NRAS mutations as predictive biomarkers for single agent panitumumab (pmab) response in a randomized, phase III metastatic colorectal cancer (mCRC) study (20020408). J Clin Oncol 31 (Suppl. abstr 3617)

8. Douillard JY, Siena S, Cassidy J, et al (2010). Randomized, phase III trial of panitumumab with infusional fluorouracil, leucovorin, and oxaliplatin (FOLFOX4) versus FOLFOX4 alone as first-line treatment in patients with previously untreated metastatic colorectal cancer: the PRIME study. J Clin Oncol 28:4697-705

9. Bokemeyer C, Van Cutsem E, Rougier P, et al (2012). Addition of cetuximab to chemotherapy as first-line treatment for KRAS wild-type metastatic colorectal cancer: pooled analysis of the CRYSTAL and OPUS randomised clinical trials. Eur J Cancer 48:1466-75

10. Stintzing S, Jung A, Rossius L, et al (2013). Analysis of KRAS/ NRAS and BRAF mutations in FIRE-3: A randomized phase III study of FOLFIRI plus cetuximab or bevacizumab as first-line treatment for wild-type KRAS (exon 2) metastatic colorectal cancer patients. Eur J Cancer 49 (suppl.3 LBA17) 